Pacific

Journal of

Mathematics

LOOP SPACES OF $H$-SPACES WITH FINITELY

GENERATED COHOMOLOGY

Yusuke KaWAMOTo 


\title{
LOOP SPACES OF $H$-SPACES WITH FINITELY GENERATED COHOMOLOGY
}

\author{
Yusuke KaWAMOTO
}

\begin{abstract}
Suppose $X$ is a simply connected $\bmod p \boldsymbol{H}$-space such that the $\bmod p$ cohomology $H^{*}(\Omega X)$ is a finitely generated algebra. We show that the loop space $\Omega X$ is homotopy equivalent to a finite product of Eilenberg-MacLane spaces $K(\mathbb{Z}, 1), K(\mathbb{Z}, 2)$ and $K\left(\mathbb{Z} / p^{i}, 1\right)$ for $i \geq 1$. This is a generalization of the result due to Lin, in which the same result was proved under the assumption that $X$ is an $A_{p}$-space.
\end{abstract}

\section{Introduction.}

Let $p$ be an odd prime. We assume that all spaces are completed at $p$ in the sense of Bousfield-Kan [2], and the cohomologies are taken with $\mathbb{Z} / p$ coefficients unless otherwise specified. In this paper, we investigate the homotopy type for the loop space of an $H$-space whose cohomology is finitely generated as an algebra. In the case of the cohomology is finite dimensional, there is the following theorem due to Aguadé-Smith:

Theorem $1.1([\mathbf{1}])$. If $X$ is a simply connected mod $p H$-space such that $H^{*}(\Omega X)$ is finite dimensional, then $\Omega X$ has the homotopy type of a torus.

The above theorem is known as the mod $p$ torus theorem, and some generalizations of Theorem 1.1 are investigated by Hemmi [8] and McGibbon [15]. Hemmi showed that a connected finite quasi $C_{p^{-}}$-space has the homotopy type of a torus, where a quasi $C_{p}$-space is defined as an $H$-space which has certain higher homotopy associativity and commutativity (see [8, Def. $2.1])$.

Our main result is stated as follows:

Theorem A. If $X$ is a simply connected mod $p H$-space such that $H^{*}(\Omega X)$ is finitely generated as an algebra, then $\Omega X$ is homotopy equivalent to a finite product of Eilenberg-MacLane spaces $K(\mathbb{Z}, 1), K(\mathbb{Z}, 2)$ and $K\left(\mathbb{Z} / p^{i}, 1\right)$ for $i \geq 1$.

Theorem A generalizes Theorem 1.1 since $K(\mathbb{Z}, 2)$ and $K\left(\mathbb{Z} / p^{i}, 1\right)$ for $i \geq 1$ do not have the finite cohomology. Our theorem also generalizes a result of Lin [12] who has shown Theorem A under the assumption that $X$ is an $A_{p}$-space in the sense of Stasheff [19]. We owe much to the results 
in [12] and [13] (see $\S 2$ ). From the result of Hemmi, it may be possible to generalize our result to the case of quasi $C_{p}$-spaces instead of loop spaces on $H$-spaces.

For $p=2$, there is the following more general result due to Slack and Broto-Crespo:

Theorem 1.2 ([18, Cor. 0.2], [3, Cor. 1.5]). If $X$ is a connected homotopy commutative mod $2 \mathrm{H}$-space such that the mod 2 cohomology $H^{*}(X)$ is finitely generated as an algebra, then $X$ is homotopy equivalent to a finite product of Eilenberg-MacLane spaces $K(\mathbb{Z}, 1), K(\mathbb{Z}, 2)$ and $K\left(\mathbb{Z} / 2^{i}, 1\right)$ for $i \geq 1$.

We remark that for the odd prime case, the corresponding result of Theorem 1.2 does not hold. In fact, Iriye-Kono [9] have shown that for an odd prime $p$, any mod $p H$-space possesses a multiplication which is homotopy commutative. Moreover, one may guess that a homotopy commutative $\bmod p$ loop space which has the finitely generated cohomology is homotopy equivalent to a product of Eilenberg-MacLane spaces. However, we note that $S p(2)$ for $p=3$ and $S^{3}$ for $p \geq 5$ are counterexamples (see [14, Thm. 2]).

In the proof of Theorem A, we use a technique for $H$-fibrations introduced by Broto-Crespo [3]. Their observation was concentrated on the mod 2 case, and some parts of their proof have generalizations to the odd prime cases with simple modifications (see Proposition 3.3 and Proposition 3.6). We combine these results with the computations in $\S 2$ for the cohomology of $\Omega X$ to establish a proof of Theorem A (see $\S 4$ ).

Now we provide an outline of the proof of Theorem A so that the reader has an overview of the ideas and strategy.

For a mod $p H$-space $X$ satisfying the assumption, we consider the threeconnected cover $\tilde{X}$. Then we have a fibration

$$
\Omega \tilde{X} \longrightarrow \Omega X \longrightarrow K,
$$

where $K$ is a finite product of Eilenberg-MacLane spaces of degrees 1 and 2. We see that $H^{*}(\Omega \tilde{X})$ is free commutative, finitely generated as an algebra which has generators in degrees $2 p, 2 p+1,2 p^{2}$ and $2 p^{2}+1$ with certain Steenrod relations induced from $H^{*}(\Omega X)$ (see Proposition 2.3). For a generator $x$ of degree $2 p$, using the Lannes theory, we construct an $H$-map $\phi: B \mathbb{Z} / p \rightarrow \Omega \tilde{X}$ such that $\phi^{*}(x)=\omega^{p}$, where $\omega \in H^{2}(B \mathbb{Z} / p)$ denotes the generator. We construct an $H$-fibration

$$
B \mathbb{Z} / p \stackrel{\phi}{\longrightarrow} \Omega \tilde{X} \longrightarrow E_{1}
$$

where $E_{1}$ is an $H$-space given by the Borel construction for $\phi$. By repeating this construction, we have a sequence of $H$-spaces and $H$-maps

$$
\Omega \tilde{X} \longrightarrow E_{1} \longrightarrow E_{2} \longrightarrow \cdots,
$$


and if we set $Y=\underline{\lim }_{s} E_{s}$, then the three-connected cover $Y\langle 3\rangle \simeq \Omega \tilde{X}$, and the cohomology $H^{*}(Y)$ is related to $H^{*}(\Omega \tilde{X})$ in that $H^{*}(Y)$ has an additional three dimensional generator and one less $2 p$-dimensional generators (see Proposition 4.1). Applying this procedure a finite number of times, we obtain a $\bmod p H$-space $Z$ such that $Z\langle 3\rangle \simeq \Omega \tilde{X}$ and the cohomology $H^{*}(Z)$ has no $2 p$-dimensional generator.

By using the same methods, we can knock off the $2 p^{2}$-dimensional generators, and thus we obtain a mod $p H$-space $W$ such that $W\langle 3\rangle \simeq \Omega \tilde{X}$ and the cohomology $H^{*}(W)$ is an exterior algebra with generators in degrees 3 and $2 p+1$ (see Proposition 4.8).

By the localization theory due to Dror Farjoun and Neisendorfer, we can show that $W$ is also the loop space on an $H$-space, and so $W$ is contractible by Theorem 1.1. This implies that $\Omega \tilde{X}$ is also contractible and therefore $\Omega X \simeq K$. The ideas and strategy come from [3].

This paper is organized as follows: In $\S 2$, we prove Theorem A using Theorem 1.1, Proposition 2.6 and results for the localization theory due to Dror Farjoun [6] and Neisendorfer [17]. Here Proposition 2.6 is the key to the proof of Theorem A, and we postpone the proof until $\S 4$. In $\S 3$, we recall the Lannes theory and show some properties for $H$-fibrations. In $\S 4$, we prove Proposition 2.6 using the results of $\S 3$.

The author would like to thank Prof. Y. Hemmi, Prof. K. Ishiguro and Prof. M. Imaoka for their many helpful suggestions and conversations. We also appreciate the referee for many useful comments.

\section{Proof of Theorem A.}

In this section we prove Theorem A. Thus, throughout this section, the space $X$ is always assumed to satisfy the hypothesis of Theorem A. First, we recall the following result due to Lin:

Theorem 2.1 ([12, Thm. A $]) \cdot H^{*}(\Omega X)$ is free commutative, primitively generated on generators in degrees $1,2,2 p, 2 p+1,2 p^{2}$ and $2 p^{2}+1$.

Remark 2.2. Lin has proved that if $X$ is a simply connected mod $p H$ space such that $H^{*}(\Omega X)$ is finitely generated as an algebra, then $H^{*}(\Omega X)$ is primitively generated on generators in degrees $1,2,2 p, 2 p+1,2 p^{2}$ and $2 p^{2}+1$, and under the assumption that $H_{*}(X)$ is associative, $H^{*}(\Omega X)$ is free commutative. We note that his proof does not use this assumption to show that $H^{*}(\Omega X)$ is primitively generated (see [12, Cor. 2.2, Thm. 2.3]). But we see that the last statement also holds without this assumption. In fact, since $\Omega X$ is homotopy associative, homotopy commutative $H$-space and $H^{*}(\Omega X)$ is primitively generated, by a theorem of Browder [4, Thm. 8.15], $H^{*}(\Omega X)$ is free commutative. 
By [12, Cor. 1.2, Thm. 2.1], $H^{*}(X)$ is generated by odd degree generators in degrees $2 p^{j}+1$ for some $j \geq 0$ and even degree generators in degrees 2 , $2 p^{j}+2$ for some $j \geq 1$. We choose the basis of $H^{2}(X)$ and $H^{3}(X)$ as $B_{2}=\left\{x_{j_{0}}\right\} \cup\left\{x_{j_{1}}\right\} \cup \cdots \cup\left\{x_{j_{r}}\right\}$ and $B_{3}=\left\{\beta_{1}\left(x_{j_{1}}\right)\right\} \cup \cdots \cup\left\{\beta_{r}\left(x_{j_{r}}\right)\right\} \cup\left\{y_{k}\right\}$, respectively, where $x_{j_{0}}$ are the mod $p$ reductions of the integral classes and $\beta_{s}$ denotes the $s$-th Bockstein operation. We define a generalized EilenbergMacLane space $K$ as

$$
K=\prod_{\left\{x_{j_{0}}\right\}} K(\mathbb{Z}, 2) \times \prod_{\left\{x_{j_{1}}\right\}} K(\mathbb{Z} / p, 2) \times \cdots \times \prod_{\left\{x_{j_{r}}\right\}} K\left(\mathbb{Z} / p^{r}, 2\right) \times \prod_{\left\{y_{k}\right\}} K(\mathbb{Z}, 3) .
$$

Let $f: X \rightarrow K$ be an $H$-map which represents the generators of the integral cohomology of dimension 2 and 3 , and $\tilde{X}$ denote the homotopy fiber of $f$. Then, $\tilde{X}$ is an $H$-space and $2 p$-connected. By the spectral sequence argument, we see that $H^{*}(\Omega \tilde{X})$ is finitely generated as an algebra, and so $\tilde{X}$ satisfies the same conditions as $X$.

Now we define an algebra $A$ as

$$
A=\mathbb{Z} / p\left[x_{1}, \ldots, x_{m}, y_{1}, \ldots, y_{n}\right] \otimes \Lambda\left(z_{1}, \ldots, z_{m+n}, w_{1}, \ldots, w_{n}\right),
$$

where $\left|x_{i}\right|=2 p$ for $1 \leq i \leq m,\left|y_{j}\right|=2 p^{2}$ for $1 \leq j \leq n,\left|z_{k}\right|=2 p+1$ for $1 \leq k \leq m+n$, and $\left|w_{l}\right|=2 p^{2}+1$ for $1 \leq l \leq n$.

Then we can prove the following proposition:

Proposition 2.3. $H^{*}(\Omega \tilde{X}) \cong A$ as algebras, and the following operations act on $H^{*}(\Omega \tilde{X})$ :

$$
\begin{cases}\beta\left(x_{i}\right)=z_{i} & \text { for } 1 \leq i \leq m, \\ \beta\left(y_{j}\right)=\mathcal{P}^{p}\left(z_{m+j}\right)=w_{j} & \text { for } 1 \leq j \leq n .\end{cases}
$$

For a $\bmod p H$-space $Y$, we denote the primitive and indecomposable modules of the Hopf algebra $H^{*}(Y)$ by $P H^{*}(Y)$ and $Q H^{*}(Y)$, respectively. We need the following fact for the proof of Proposition 2.3.

Lemma 2.5 ([16, Thm. 4.21]). If $Y$ is a connected mod $p H$-space, then there is the following exact sequence:

$$
0 \rightarrow P\left(\xi H^{*}(Y)\right) \rightarrow P H^{*}(Y) \rightarrow Q H^{*}(Y),
$$

where $\xi: H^{*}(Y) \rightarrow H^{*}(Y)$ is a map defined as $\xi(x)=x^{p}$.

Proof of Proposition 2.3. By Theorem 2.1, $H^{*}(\Omega \tilde{X})$ is free commutative, and has generators $x_{i}, y_{j}, z_{k}$ and $w_{l}$ with $\left|x_{i}\right|=2 p,\left|y_{j}\right|=2 p^{2},\left|z_{k}\right|=2 p+1$ and $\left|w_{l}\right|=2 p^{2}+1$ for $1 \leq i \leq m, 1 \leq j \leq n, 1 \leq k \leq q$ and $1 \leq l \leq r$, where generators are primitive.

Since $\beta\left(x_{i}\right) \in P H^{2 p+1}(\Omega \tilde{X})$ for $1 \leq i \leq m$, we see that $\beta\left(x_{i}\right) \in$ $Q H^{2 p+1}(\Omega \tilde{X})$ by Lemma 2.5. By [13, Cor. E], we have that $\beta\left(x_{i}\right) \neq 0$, and if $i_{1} \neq i_{2}$, then $\beta\left(x_{i_{1}}\right) \neq \beta\left(x_{i_{2}}\right)$. Thus, we can set $\beta\left(x_{i}\right)=z_{i}$ for $1 \leq i \leq m$. Similarly, we can set $\beta\left(y_{j}\right)=w_{j}$ for $1 \leq j \leq n$. 
Since the suspension map $\sigma^{*}: Q H^{2 p^{2}+2}(\tilde{X}) \rightarrow P H^{2 p^{2}+1}(\Omega \tilde{X})$ is an epimorphism, and $\beta: Q H^{2 p^{2}+1}(\tilde{X}) \rightarrow Q H^{2 p^{2}+2}(\tilde{X})$ is also an epimorphism by [12, Thm. 1.10], we have that $w_{l} \in \beta P H^{2 p^{2}}(\Omega \tilde{X})$ for $1 \leq l \leq r$. Thus, we have that $w_{l} \in \beta Q H^{2 p^{2}}(\Omega \tilde{X})$ by Lemma 2.5, which implies that $r=n$.

Using [12, Thm. 1.9], the similar arguments show that $w_{l} \in$ $\mathcal{P}^{p} Q H^{2 p+1}(\Omega \tilde{X})$ for $1 \leq l \leq n$. We can assume that $\mathcal{P}^{p}\left(z_{m+l}\right)=w_{l}$ for $1 \leq l \leq n$ since $\mathcal{P}^{p}\left(z_{k}\right)=\mathcal{P}^{1} \beta \mathcal{P}^{p-1}\left(x_{k}\right)+\beta \mathcal{P}^{p}\left(x_{k}\right)=0$ for $1 \leq k \leq m$.

If we set

$$
\mathcal{P}^{p}\left(z_{m+n+1}\right)=\sum_{l=1}^{n} \sigma_{l} w_{l}
$$

for $\sigma_{l} \in \mathbb{Z} / p$, then for

$$
\zeta=z_{m+n+1}-\sum_{l=1}^{n} \sigma_{l} z_{m+l},
$$

we have that $\mathcal{P}^{p}(\zeta)=0$. Since $\sigma^{*}: Q H^{2 p+2}(\tilde{X}) \rightarrow P H^{2 p+1}(\Omega \tilde{X})$ is an epimorphism, $\zeta=\sigma^{*}(\mu)$ for some $\mu \in Q H^{2 p+2}(\tilde{X})$. Since $\sigma^{*}\left(\mathcal{P}^{p}(\mu)\right)=$ $\mathcal{P}^{p}(\zeta)=0$, by $\left[\mathbf{1 0}\right.$, Thm. B], there exists $\nu \in Q H^{2 p+1}(\tilde{X})$ such that $\mathcal{P}^{p}(\mu)=$ $\beta \mathcal{P}^{p}(\nu)$ in $Q H^{2 p^{2}+2}(\tilde{X})$. Applying the Adem relation $\mathcal{P}^{p} \beta=\mathcal{P}^{1} \beta \mathcal{P}^{p-1}+\beta \mathcal{P}^{p}$ to $\nu$, we have that $\mathcal{P}^{p}(\mu)=\mathcal{P}^{p}(\beta(\nu))$, which implies that $\mu=\beta(\nu)$ by $[\mathbf{1 2}$, Thm. 1.9]. Then, $\zeta=\sigma^{*}(\mu)=\beta\left(\sigma^{*}(\nu)\right) \in \beta Q H^{2 p}(\Omega \tilde{X})$ by Lemma 2.5, which implies that

$$
\zeta=\sum_{k=1}^{m} \tau_{k} z_{k}
$$

for $\tau_{k} \in \mathbb{Z} / p$. Therefore, we have that

$$
z_{m+n+1}=\sum_{k=1}^{m} \tau_{k} z_{k}+\sum_{l=1}^{n} \sigma_{l} z_{m+l},
$$

which implies that $q=m+n$. This completes the proof.

The following proposition is crucial for our study, which will be proved in $\S 4$ using the Lannes theory.

Proposition 2.6. If $Y$ is a $\bmod p H$-space with $H^{*}(Y) \cong A$ as algebras, and the operations (2.4) act on $H^{*}(Y)$, then there is a simply connected $\bmod p$ finite $H$-space $W$ such that $Y \simeq W\langle 3\rangle$, where $W\langle 3\rangle$ is the threeconnected cover of $W$.

Using Proposition 2.6, we can prove Theorem A as follows:

Proof of Theorem A. By Proposition 2.3 and Proposition 2.6, there exists a simply connected $\bmod p$ finite $H$-space $W$ such that $\Omega \tilde{X} \simeq W\langle 3\rangle$. Let $L_{g}$ denote the localization functor with respect to a map $g$ constructed by 
Dror Farjoun [6]. For the constant map $c: B \mathbb{Z} / p \rightarrow *, L_{c}(\Omega \tilde{X}) \simeq W$ by the results due to Neisendorfer $\left[\mathbf{1 7}\right.$, Thm. 0.1]. Since $L_{c}(\Omega \tilde{X}) \simeq \Omega L_{\Sigma c}(\tilde{X})$ by [6, Thm. 3.A.1], and $L_{\Sigma c}$ preserves the $H$-structure, we see that the space $W$ is the loop space of an $H$-space. By Theorem 1.1, $W$ is contractible, and so $\Omega \tilde{X} \simeq W\langle 3\rangle$ is also contractible. Therefore, $\Omega X \simeq \Omega K$, and we have the required conclusion. This completes the proof of Theorem A.

\section{Lannes $T$-functor and $H$-fibrations.}

In this section we recall some results concerning the Lannes theory and the $H$-fibrations, which will be used in the next section.

Let $\mathcal{K}$ denote the category of unstable $\mathcal{A}_{p}$-algebras. The objects of $\mathcal{K}$ are called $\mathcal{K}$-algebras. It is known that $H^{*}(X)$ is a $\mathcal{K}$-algebra for any space $X$.

The Lannes $T$-functor $T: \mathcal{K} \rightarrow \mathcal{K}$ is a left adjoint of the functor $H^{*}(B \mathbb{Z} / p) \otimes-$, that is, there is the adjoint isomorphism $\operatorname{Hom}_{\mathcal{K}}(T(A), B) \cong$ $\operatorname{Hom}_{\mathcal{K}}\left(A, H^{*}(B \mathbb{Z} / p) \otimes B\right)$ for $\mathcal{K}$-algebras $A$ and $B$.

For a $\mathcal{K}$-map $f: A \rightarrow H^{*}(B \mathbb{Z} / p)$, its adjoint restricts to a $\mathcal{K}$-map $T(A)^{0} \rightarrow \mathbb{Z} / p$, where $T(A)^{0}$ is the subalgebra of $T(A)$ of elements of degree 0 . The connected component of $T(A)$ corresponding to $f$ is defined by $T_{f}(A)=T(A) \otimes_{T(A)^{0}} \mathbb{Z} / p$, and there is the natural $\mathcal{K}$-map $\epsilon_{f}: A \rightarrow T_{f}(A)$.

The evaluation map $e: B \mathbb{Z} / p \times \operatorname{Map}(B \mathbb{Z} / p, X) \rightarrow X$ induces a $\mathcal{K}$ map $e^{*}$, and taking the adjoint of this yields a $\mathcal{K}$-map $\lambda: T\left(H^{*}(X)\right) \rightarrow$ $H^{*}(\operatorname{Map}(B \mathbb{Z} / p, X))$. On the component level, for a map $\phi: B \mathbb{Z} / p \rightarrow X$, there is a $\mathcal{K}$-map $\lambda_{\phi^{*}}: T_{\phi^{*}}\left(H^{*}(X)\right) \rightarrow H^{*}\left(\operatorname{Map}(B \mathbb{Z} / p, X)_{\phi}\right)$. The composite $\lambda_{\phi^{*}} \epsilon_{\phi^{*}}$ is induced by the evaluation at the base point $e_{\phi}: \operatorname{Map}(B \mathbb{Z} / p, X)_{\phi} \rightarrow$ $X$. The following theorem is due to Lannes:

Theorem 3.1 ([11, Thm. 3.2.1]). Let $X$ be a space and $\phi: B \mathbb{Z} / p \rightarrow X$ be a map. If $T_{\phi^{*}}\left(H^{*}(X)\right)^{1}=0$, then $\lambda_{\phi^{*}}: T_{\phi^{*}}\left(H^{*}(X)\right) \rightarrow H^{*}\left(\operatorname{Map}(B \mathbb{Z} / p, X)_{\phi}\right)$ is an isomorphism.

For the cohomology of an $H$-space, Dwyer-Wilkerson have proved the following:

Proposition 3.2 ([7, Thm. 3.2, Lemma 4.5]). If $X$ is a mod $p H$-space with finitely generated cohomology and $f: H^{*}(X) \rightarrow H^{*}(B \mathbb{Z} / p)$ is a $\mathcal{K}$ map, then $\epsilon_{f}: H^{*}(X) \rightarrow T_{f}\left(H^{*}(X)\right)$ is an isomorphism.

Recently, an important theory of $H$-fibrations using the Lannes theory was introduced by Broto-Crespo [3]. Their observation was concentrated on the mod 2 case. However, we also have the corresponding results for the odd prime case.

Proposition 3.3. Let $X$ be a mod $p H$-space with finitely generated cohomology, and $\phi: B \mathbb{Z} / p \rightarrow X$ be an $H$-map with $H^{*}(B \mathbb{Z} / p)$ is finitely 
generated $H^{*}(X)$-module induced by $\phi^{*}$. If

$$
B \mathbb{Z} / p \stackrel{\phi}{\longrightarrow} X \stackrel{\psi}{\longrightarrow} Y
$$

is a principal fibration, then $Y$ is an $H$-space and $\psi$ is an $H$-map.

Lemma 3.4. Let $c: B \mathbb{Z} / p \times B \mathbb{Z} / p \rightarrow Y$ denote the constant map, where $Y$ comes from Proposition 3.3. Then the base point evaluation map $e_{c}$ : $\operatorname{Map}(B \mathbb{Z} / p \times B \mathbb{Z} / p, Y)_{c} \rightarrow Y$ is a homotopy equivalence.

Proof. We have the following commutative diagram of fibrations:

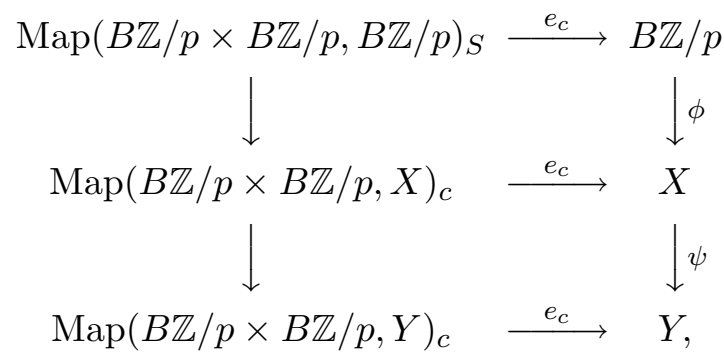

where $S=\{g: B \mathbb{Z} / p \times B \mathbb{Z} / p \rightarrow B \mathbb{Z} / p \mid \phi g \simeq c\}$ and $e_{c}$ denote the base point evaluation maps.

Since $X$ has the finitely generated cohomology, $e_{c}: \operatorname{Map}(B \mathbb{Z} / p \times B \mathbb{Z} / p, X)_{c}$ $\rightarrow X$ is a homotopy equivalence by [7, Thm. 3.2]. It is known that $H^{*}(B \mathbb{Z} / p) \cong \Lambda(\theta) \otimes \mathbb{Z} / p[\omega]$ with $\beta(\theta)=\omega$. For a map $g: B \mathbb{Z} / p \times B \mathbb{Z} / p \rightarrow$ $B \mathbb{Z} / p$ with $\phi g \simeq c$, there exists some $n \geq 1$ so that $g^{*}(\omega)^{n}=g^{*}\left(\omega^{n}\right)=0$ since $H^{*}(B \mathbb{Z} / p)$ is finitely generated $H^{*}(X)$-module induced by $\phi^{*}$ and $g^{*} \phi^{*}=0$, which implies that $g^{*}(\omega)=0$. If we put $g^{*}(\theta)=a_{1} \theta_{1}+a_{2} \theta_{2}$ for $a_{1}, a_{2} \in \mathbb{Z} / p$, then $g^{*}(\omega)=\beta\left(g^{*}(\theta)\right)=a_{1} \omega_{1}+a_{2} \omega_{2}=0$, and we must have $a_{1}=a_{2}=0$, which implies that $g^{*}(\theta)=0$. By a result of Lannes [11, Thm. 3.1.1], we obtain that $g \simeq c$.

Then we have that $S=\{c\}$, and thus $e_{c}: \operatorname{Map}(B \mathbb{Z} / p \times B \mathbb{Z} / p, B \mathbb{Z} / p)_{S} \rightarrow$ $B \mathbb{Z} / p$ is a homotopy equivalence. Using the five lemma, $e_{c}: \operatorname{Map}(B \mathbb{Z} / p \times$ $B \mathbb{Z} / p, Y)_{c} \rightarrow Y$ is a homotopy equivalence, and thus we have the required conclusion.

For the proof of Proposition 3.3, we need the following fact which is known as the Zabrodsky lemma:

Lemma 3.5 ([21, Lemma 3.1]). Let

$$
F \stackrel{i}{\longrightarrow} E \stackrel{p}{\longrightarrow} B
$$

be a principal fibration, and $Y$ be a space which satisfies that $e_{c}: \operatorname{Map}(F, Y)_{c}$ $\rightarrow Y$ is a homotopy equivalence. Then the induced map $\operatorname{Map}(B, Y) \rightarrow$ $\operatorname{Map}(E, Y)_{S}$ is a homotopy equivalence, where $S=\{g: E \rightarrow Y \mid g i \simeq c\}$.

Now we can prove Proposition 3.3 as follows: 
Proof of Proposition 3.3. By Lemma 3.4, the evaluation map $e_{c}$ : $\operatorname{Map}(B \mathbb{Z} / p \times B \mathbb{Z} / p, Y)_{c} \rightarrow Y$ is a homotopy equivalence. Then, applying Lemma 3.5 to a principal fibration

$$
B \mathbb{Z} / p \times B \mathbb{Z} / p \stackrel{\phi \times \phi}{\longrightarrow} X \times X \stackrel{\psi \times \psi}{\longrightarrow} Y \times Y,
$$

we have that $\operatorname{Map}(Y \times Y, Y) \simeq \operatorname{Map}(X \times X, Y)_{S}$, where $S=\{g: X \times X \rightarrow$ $Y \mid g(\phi \times \phi) \simeq c\}$. If we denote the multiplication of the $H$-space $X$ as $\mu_{X}$, then there is a map $\mu_{Y}: Y \times Y \rightarrow Y$ so that $\psi \mu_{X} \simeq \mu_{Y}(\psi \times \psi)$. Using Lemma 3.5 again, we see that the map $\mu_{Y}$ gives an $H$-structure on $Y$. This completes the proof.

Proposition 3.6. Suppose that there is an $H$-fibration

$$
B \mathbb{Z} / p \stackrel{\phi_{i}}{\longrightarrow} X_{i} \stackrel{\psi_{i}}{\longrightarrow} X_{i+1}
$$

for $i \geq 0$, and we put $Y=\lim _{i} X_{i}$. If $H^{*}(Y)$ is finitely generated as an algebra, then the space $Y$ has an $H$-structure.

Proof. We set $\mu=\lim _{i} \mu_{i}: Y \times Y \rightarrow Y$ for the multiplication $\mu_{i}: X_{i} \times X_{i} \rightarrow$ $X_{i}$ of the $H$-space $X_{i}$. Let $\iota_{j}: Y \rightarrow Y \times Y$ denote the inclusion map on the $j$-th factor for $j=1,2$. If we show that $\mu \iota_{j} \simeq 1_{Y}$ for $j=1,2$, then we have the required conclusion.

We denote the inclusion map as $\kappa_{i}: X_{i} \rightarrow Y$ for $i \geq 0$. Since $\mu_{i}$ is a multiplication for $i \geq 0$, we have that $\mu \iota_{j} \kappa_{i} \simeq \kappa_{i} \mu_{i} \iota_{j}^{i} \simeq \kappa_{i}$, where $\iota_{j}^{i}: X_{i} \rightarrow$ $X_{i} \times X_{i}$ denotes the inclusion map on the $j$-th factor for $j=1,2$. By [20, Prop. 4], the obstruction to construct a homotopy between $\mu \iota_{j}$ and $1_{Y}$ lies in

$$
\varliminf_{i}^{k} \pi_{k}\left(\operatorname{Map}\left(X_{i}, Y\right)_{\kappa_{i}}\right)
$$

for $k \geq 1$. Since $H^{*}(Y)$ is a finitely generated algebra, $\operatorname{Map}(B \mathbb{Z} / p, Y)_{c} \simeq Y$ by [7, Thm. 3.2]. Then, applying Lemma 3.5 to the fibration (3.7), we have that $\operatorname{Map}\left(X_{i}, Y\right)_{\kappa_{i}} \simeq \operatorname{Map}\left(X_{i+1}, Y\right)_{\kappa_{i+1}}$ for $i \geq 0$, and so the obstruction group (3.8) vanishes. This completes the proof.

Now we introduce a result which is useful to compute the Serre spectral sequence for an $H$-fibration, which will be used in $\S 4$. Let $X$ and $Y$ be $H$-spaces and

$$
X \longrightarrow Y \longrightarrow B^{2} \mathbb{Z} / p
$$

be an $H$-fibration. We consider the Serre spectral sequence for the fibration whose $E_{2}$-term is given as

$$
E_{2}^{*, *}=H^{*}\left(B^{2} \mathbb{Z} / p\right) \otimes H^{*}(X) .
$$

Then we see that the spectral sequence has a differential Hopf algebra structure, and for $r \geq 2$, if we put $A_{r}=E_{r}^{*, 0}$ and $B_{r}=E_{r}^{0, *}$, then they have Hopf algebra structures induced from the $E_{2}$-term. 
Proposition 3.10. (1) If $d_{r}\left(B_{r}\right) \neq 0$, then the transgression $\tau: B_{r}^{r-1} \rightarrow$ $P^{r}\left(A_{r}\right)$ is non-trivial.

(2) For $x \in B_{r}^{q}, d_{r}(x) \in P^{r}\left(A_{r}\right) \otimes B_{r}^{q-r+1}$, where $P^{n}\left(A_{r}\right)$ denotes the primitive module of $A_{r}^{n}$.

We need the following lemma to show Proposition 3.10:

Lemma 3.11. (1) For $r \geq 2$, the $E_{r}$-term is given as

$$
E_{r}^{*, *} \cong A_{r} \otimes B_{r} \otimes \Lambda\left(\alpha_{1}, \ldots, \alpha_{k}\right),
$$

where $\alpha_{i} \in E_{r}^{s_{i}, t_{i}}$ with $s_{i}<r$ and $\left|\alpha_{i}\right|=2 m_{i}+1$ with $p \mid m_{i}$ for $1 \leq i \leq k$.

(2) If $x \in P^{2 s}\left(A_{r}\right)$ with $2 s \geq r$, then $x$ has the infinite height.

Proof. We show (1) and (2) by induction. For $r=2$, by (3.9) and since $H^{*}\left(B^{2} \mathbb{Z} / p\right)$ is free commutative, the results (1) and (2) hold. We assume that the results (1) and (2) have already shown for the $E_{r}$-term.

By a result of Browder [5, Thm. 5.8], the $E_{r+1}$-term is described as

$$
E_{r+1}^{*, *} \cong A_{r+1} \otimes B_{r+1} \otimes \Lambda\left(\alpha_{1}, \ldots, \alpha_{k}, \beta_{1}, \ldots, \beta_{l}\right),
$$

where $\alpha_{i}$ are elements stated in the lemma, $\beta_{j} \in E_{r+1}^{s_{j}, t_{j}}$ with $t_{j}<r-1$ and $\left|\beta_{j}\right|=2 n_{j}-1$ with $p \mid n_{j}$ for $1 \leq j \leq l$. From the proof of [5, Thm. 5.8], we see that $\beta_{j}=\left\{x_{j} \cdot d_{r}\left(x_{j}\right)^{p-1}\right\}$ for some $x_{j} \in E_{r}^{*, *}$ with $d_{r}\left(x_{j}\right) \in P\left(A_{r}\right)$. But by assumption, $d_{r}\left(x_{j}\right)$ has the infinite height, and so the element of the form $\beta_{j}$ cannot occur, which shows (1).

For a non-trivial element $x \in P^{2 s}\left(A_{r+1}\right)$ with $2 s \geq r+1$, we assume that $x^{p^{k}}=0$ for some $k \geq 1$, and obtain a contradiction from this assumption. By inductive hypothesis, $x^{p^{k}} \neq 0 \in A_{r}$, and then there exists an element $y \in E_{r}^{*, *}$ so that $d_{r}(y)=x^{p^{k}}$. By the form of the $E_{r}$-term, we have either a generator $z \in B_{r}^{r-1}$ with $d_{r}(z)=x^{p^{k_{1}}}$ for some $k_{1} \leq k$ or a generator $\alpha \in E_{r}^{*, *}$ with $d_{r}(\alpha)=x^{p^{k_{2}}}$ for some $k_{2} \leq k$. On the one hand, if $d_{r}(z)=$ $x^{p^{k_{1}}}$, then $\left|x^{p^{k_{1}}}\right|=r<|x|$, which causes a contradiction. On the other hand, if $d_{r}(\alpha)=x^{p^{k_{2}}}$, then $\left|x^{p^{k_{2}}}\right|=2 m+2$ for some $m \geq 1$ with $p \mid m$. This shows that $k_{2}=0$, and so $\{x\}=0$ in the $E_{r+1}$-term, which also causes a contradiction. This completes the proof.

Now we can prove Proposition 3.10 as follows:

Proof of Proposition 3.10. First we show (1). By assumption, there is an element $x \in B_{r}^{q}$ so that $d_{r}(x) \neq 0$. We can assume that if $y \in B_{r}^{\bar{q}}$ with $\bar{q}<q$, then $d_{r}(y)=0$. If we set that

$$
\Delta(x)=x \otimes 1+1 \otimes x+\sum_{i} \bar{x}_{i} \otimes \overline{\bar{x}}_{i}
$$

then $\Delta\left(d_{r}(x)\right)=d_{r}(\Delta(x))=d_{r}(x) \otimes 1+1 \otimes d_{r}(x)$, and so $d_{r}(x) \in P\left(E_{r}^{r, q-r+1}\right)$. By Lemma 3.11, the primitive elements of $E_{r}^{*, *}$ consist of $P\left(A_{r}\right), P\left(B_{r}\right)$ and 
$\alpha_{i} \in E_{r}^{s, t}$ with $s<r$, and then we have that $q=r-1$ and $d_{r}(x) \in P^{r}\left(A_{r}\right)$. This implies the required conclusion.

Next to show (2), let $x \in B_{r}^{q}$. By Lemma 3.11, we can set that

$$
d_{r}(x)=\sum_{i \geq 1} a_{i} \cdot b_{i} \in E_{r}^{r, q-r+1},
$$

where $a_{i} \in A_{r} \otimes \Lambda\left(\alpha_{1}, \ldots, \alpha_{k}\right), b_{i} \in B_{r}$ with $\left|a_{i}\right|+\left|b_{i}\right|=q+1$ for $i \geq 1$. We can assume that the elements $b_{i}$ are linearly independent for $i \geq 1$. Then we have that

$$
\begin{aligned}
\Delta\left(d_{r}(x)\right)= & \sum_{i} \Delta\left(a_{i}\right) \Delta\left(b_{i}\right) \\
= & \sum_{i}\left(a_{i} \otimes 1+1 \otimes a_{i}+\sum_{j} \bar{a}_{i, j} \otimes \overline{\bar{a}}_{i, j}\right) \\
& \cdot\left(b_{i} \otimes 1+1 \otimes b_{i}+\sum_{k} \bar{b}_{i, k} \otimes \overline{\bar{b}}_{i, k}\right),
\end{aligned}
$$

where $0<\left|\bar{a}_{i, j}\right|,\left|\overline{\bar{a}}_{i, j}\right|<\left|a_{i}\right|$ and $0<\left|\bar{b}_{i, k}\right|,\left|\overline{\bar{b}}_{i, k}\right|<\left|b_{i}\right|$. On the other hand, we obtain that

$$
\Delta\left(d_{r}(x)\right)=d_{r}(\Delta(x)) \in \bigoplus_{s+t=q}\left(E_{r}^{r, s-r+1} \otimes B_{r}^{t}\right) \oplus\left(B_{r}^{s} \otimes E_{r}^{r, t-r+1}\right) .
$$

For the dimensional reason, we see that $\sum_{i, j} \bar{a}_{i, j} b_{i} \otimes \overline{\bar{a}}_{i, j}=0$, which implies that $\sum_{j} \bar{a}_{i, j} \otimes \overline{\bar{a}}_{i, j}=0$ for $i \geq 1$ since $b_{i}$ are linearly independent. This implies that

$$
a_{i} \in P\left(A_{r} \otimes \Lambda\left(\alpha_{1}, \ldots, \alpha_{k}\right)\right)=P\left(A_{r}\right) \oplus\left\{\alpha_{1}, \ldots, \alpha_{k}\right\},
$$

and then $a_{i} \in P^{r}\left(A_{r}\right)$ for $i \geq 1$. Thus we can conclude that $d_{r}(x) \in$ $P^{r}\left(A_{r}\right) \otimes B_{r}^{q-r+1}$. This completes the proof.

Remark 3.12. We note that by Proposition 3.10, for $r \geq 2$, if either $P^{r}\left(A_{r}\right)=0$ or $Q^{r-1}\left(B_{r}\right)=0$, then $d_{r}\left(B_{r}\right)=0$.

\section{Proof of Proposition 2.6.}

In this section we prove Proposition 2.6, and thus we assume that $Y$ is a $\bmod$ $p H$-space such that $H^{*}(Y) \cong A$, and the operations (2.4) act on $H^{*}(Y)$.

For $1 \leq t \leq m+1$, we set an algebra $K_{t}$ as

$$
\begin{aligned}
K_{t}=\mathbb{Z} / p\left[x_{t}, \ldots, x_{m}, y_{1}, \ldots, y_{n}\right] & \\
& \otimes \Lambda\left(v_{1}, \ldots, v_{t-1}, z_{t}, \ldots, z_{m+n}, w_{1}, \ldots, w_{n}\right)
\end{aligned}
$$

with $x_{i}, y_{j}, z_{k}$ and $w_{l}$ are as in $A,\left|v_{q}\right|=3$ for $1 \leq q \leq t-1$. First, we prove the following proposition: 
Proposition 4.1. For $1 \leq t \leq m+1$, there is a $\bmod p H$-space $Y_{t}$ such that $Y_{t}\langle 3\rangle \simeq Y$ and $H^{*}\left(Y_{t}\right) \cong K_{t}$ with the following operations:

$$
\begin{cases}\beta\left(x_{i}\right)=z_{i} & \text { for } t \leq i \leq m, \\ \beta\left(y_{j}\right)=w_{j} & \text { for } 1 \leq j \leq n, \\ \mathcal{P}^{p}\left(z_{m+l}\right)=w_{l}+\delta_{l} & \text { for } 1 \leq l \leq n,\end{cases}
$$

where $\delta_{l}$ is some decomposable element of $K_{t}$ for $1 \leq l \leq n$.

For $1 \leq t \leq m$, we set an algebra $C_{t}$ as

$$
C_{t}=\mathbb{Z} / p\left[u, x_{t+1}, \ldots, x_{m}, y_{1}, \ldots, y_{n}\right]
$$

$\otimes \Lambda\left(v_{1}, \ldots, v_{t}, z_{t+1}, \ldots, z_{m+n}, w_{1}, \ldots, w_{n}\right)$

with $x_{i}, y_{j}, z_{k}, w_{l}$ and $v_{q}$ for $1 \leq q \leq t-1$ are as in $K_{t},|u|=2$ and $\left|v_{t}\right|=3$.

An algebra $A$ is said to be a $\mathcal{K}$-Hopf algebra if $A$ is a $\mathcal{K}$-algebra and has a Hopf algebra structure compatible with the $\mathcal{K}$-structure, namely the diagonal map of $A$ becomes a $\mathcal{K}$-map. It is known that for an $H$-space $X$, $H^{*}(X)$ is a $\mathcal{K}$-Hopf algebra. We see that if $K_{t}$ and $C_{t}$ have $\mathcal{K}$-Hopf algebra structures, then for the dimensional reason, $v_{q}$ is primitive for $1 \leq q \leq t$.

Lemma 4.2. Suppose that the algebras $K_{t}$ and $C_{t}$ are $\mathcal{K}$-Hopf algebras with the following operations:

$$
\begin{cases}\beta(u)=\lambda v_{t} & \text { for } \lambda=0 \text { or } 1, \\ \beta\left(x_{i}\right)=z_{i} & \text { for } t \leq i \leq m, \\ \beta\left(y_{j}\right)=w_{j} & \text { for } 1 \leq j \leq n, \\ \mathcal{P}^{p}\left(z_{m+l}\right)=w_{l}+\delta_{l} & \text { for } 1 \leq l \leq n,\end{cases}
$$

where $\delta_{l}$ is some decomposable element of $K_{t}$ for $1 \leq l \leq n$. Then the following hold:

(1) There is a map of $\mathcal{K}$-Hopf algebra $f: K_{t} \rightarrow H^{*}(B \mathbb{Z} / p)$ such that $f\left(x_{t}\right)=\omega^{p}$ and $f=0$ on the other generators of $K_{t}$, where $H^{*}(B \mathbb{Z} / p) \cong$ $\Lambda(\theta) \otimes \mathbb{Z} / p[\omega]$ with $\beta(\theta)=\omega$.

(2) There is a map of $\mathcal{K}$-Hopf algebra $g: C_{t} \rightarrow H^{*}(B \mathbb{Z} / p)$ such that $g(u)=\omega$ and $g=0$ on the other generators of $C_{t}$.

Proof. We show only (2), since (1) is proved by similar arguments.

Let $I$ denote the ideal of $C_{t}$ generated by odd degree generators. For the dimensional reason, we see that $I$ is a Hopf ideal of $C_{t}$. We show that $I$ is closed under the action of $\mathcal{A}_{p}$.

For the dimensional reason, $\mathcal{P}^{a}(I) \subset I$ for $a \geq 1$, and using the relation $\beta \beta=0$, we have that $\beta\left(z_{k}\right)=\beta\left(w_{l}\right)=0$ for $t+1 \leq k \leq m$ and $1 \leq l \leq n$. Thus, it sufficies to show that $\beta\left(v_{q}\right), \beta\left(z_{k}\right) \in I$ for $1 \leq q \leq t$ and $m+1 \leq$ $k \leq m+n$. We see that $\beta\left(v_{q}\right)$ is primitive since $v_{q}$ is primitive, and so $\beta\left(v_{q}\right)=0$ since $P^{4}\left(C_{t}\right)=0$. 
For the dimensional reason, we can put

$$
\beta\left(z_{k}\right)=\kappa u^{p+1}+\sum_{i=t+1}^{m} \rho_{i} u x_{i} \bmod I
$$

for $\kappa, \rho_{i} \in \mathbb{Z} / p$.

If $\lambda=1$, then using the relation $\beta \beta\left(z_{k}\right)=0, \kappa=\rho_{i}=0$ for $t+1 \leq i \leq m$, which implies that $\beta\left(z_{k}\right) \in I$.

When $\lambda=0$, using the relation $\beta \beta\left(z_{k}\right)=0$, we have that $\beta\left(z_{k}\right)=\kappa u^{p+1}$ $\bmod I$. For the dimensional reason, we have that $\beta\left(z_{k}\right)$ is primitive since $\beta(u)=\beta\left(v_{q}\right)=0$, which implies that $\kappa=0$, and so $\beta\left(z_{k}\right) \in I$.

From the above considerations, $C_{t} / I$ is a $\mathcal{K}$-Hopf algebra, and the quotient map $\pi: C_{t} \rightarrow C_{t} / I$ becomes a map of $\mathcal{K}$-Hopf algebra. Since $C_{t} / I$ is a polynomial algebra, there is a monomorphism of $\mathcal{K}$-Hopf algebra $\sigma: C_{t} / I \rightarrow$ $H^{*}(B V)$ by [1], where $V$ is a $(m+n-t+1)$-dimensional vector space over $\mathbb{Z} / p$. It is known that $H^{*}(B V) \cong \Lambda\left(\theta_{1}, \ldots \theta_{m+n-t+1}\right) \otimes \mathbb{Z} / p\left[\omega_{1}, \ldots \omega_{m+n-t+1}\right]$ with $\beta\left(\theta_{k}\right)=\omega_{k}$ for $1 \leq k \leq m+n-t+1$.

Taking a suitable basis of $V$, and by the argument of [1], we can assume that $\sigma(u)=\omega_{1}, \sigma\left(x_{i}\right)=\omega_{i-t+1}^{p}$ for $t+1 \leq i \leq m$, and $\sigma\left(y_{j}\right)=\omega_{m+j-t+1}^{p^{2}}$ for $1 \leq j \leq n$. If we define a map $g=(B i)^{*} \sigma \pi$, where $i: \mathbb{Z} / p \rightarrow V$ is the inclusion on the first factor, then $g$ is a map of $\mathcal{K}$-Hopf algebra which satisfies the required properties. This completes the proof.

Proof of Proposition 4.1. We proceed by an induction on $t$. For $t=1$, if we put $Y_{1}=Y$, then $Y\langle 3\rangle \simeq Y$ since $Y$ is 3 -connected, and by assumption, $H^{*}(Y) \cong A \cong K_{1}$ with the operations (2.4). Now we assume that there exists an $H$-space $Y_{t}$ with the required properties.

From now on, we construct an $H$-space $Y_{t+1}$ satisfying the required properties. For the map $f$ of Lemma 4.2, a result of Lannes [11, Thm. 3.1.1] implies that there is a map $\phi: B \mathbb{Z} / p \rightarrow Y_{t}$ such that $\phi^{*}=f$. We see that the evaluation map $e_{\phi}: \operatorname{Map}\left(B \mathbb{Z} / p, Y_{t}\right)_{\phi} \rightarrow Y_{t}$ becomes a homotopy equivalence by Theorem 3.1 and Proposition 3.2. Let $\iota: B \mathbb{Z} / p \rightarrow \operatorname{Map}\left(B \mathbb{Z} / p, Y_{t}\right)_{\phi}$ be the adjoint of $\phi \mu$, where $\mu$ is the multiplication of an $H$-structure of $B \mathbb{Z} / p$. Then we have the following commutative diagram of fibrations:

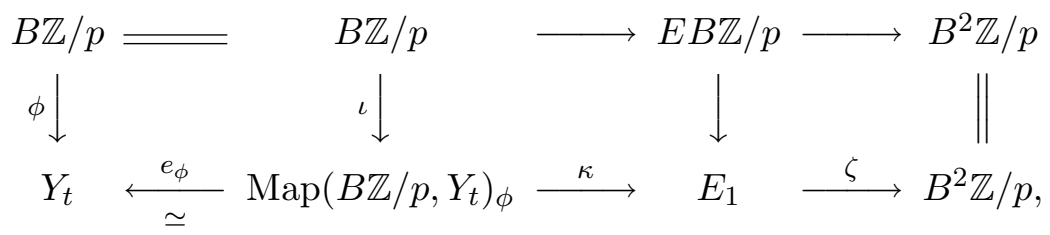

where $E_{1}=E B \mathbb{Z} / p \times_{B \mathbb{Z} / p} \operatorname{Map}\left(B \mathbb{Z} / p, Y_{t}\right)_{\phi}$ denotes the Borel construction.

Since $f$ is a map of $\mathcal{K}$-Hopf algebra, $\phi$ is an $H$-map, and so the bottom fibration becomes an $H$-fibration by Proposition 3.3. The $E_{2}$-term of the 
Serre spectral sequence for this fibration is given as

$$
E_{2}^{*, *}=H^{*}\left(B^{2} \mathbb{Z} / p\right) \otimes H^{*}\left(Y_{t}\right)
$$

for $H^{*}\left(B^{2} \mathbb{Z} / p\right) \cong \mathbb{Z} / p\left[\eta, \beta \mathcal{P}^{\Delta_{i}} \beta(\eta) \mid i \geq 0\right] \otimes \Lambda\left(\beta(\eta), \mathcal{P}^{\Delta_{i}} \beta(\eta) \mid i \geq 0\right)$, where $\mathcal{P}^{\Delta_{i}}=\mathcal{P}^{p^{i}} \ldots \mathcal{P}^{1}$ and $\eta$ denotes the fundamental class. Now we use the notations from Proposition 3.10.

For the dimensional reason and by Remark 3.12 , we have that $E_{2 p+1}^{*, *} \cong$ $E_{2}^{* * *}$. The generator $x_{i}$ is transgressive for $t \leq i \leq m$, and then by using the naturality of the diagram (4.4), and by Proposition 3.10, we obtain that

$$
d_{2 p+1}\left(x_{i}\right)= \begin{cases}\mathcal{P}^{1} \beta(\eta) & \text { for } i=t, \\ 0 & \text { for } t+1 \leq i \leq m .\end{cases}
$$

By the Kudo transgression theorem, there are the following differentials:

$$
\begin{cases}d_{2 p^{k+1}+1}\left(x_{t}^{p^{k}}\right)=\mathcal{P}^{\Delta_{k}} \beta(\eta) & \text { for } k \geq 1, \\ d_{2 p^{k}(p-1)+1}\left(\mathcal{P}^{\Delta_{k-1}} \beta(\eta) \otimes x_{t}^{p^{k-1}(p-1)}\right)=\beta \mathcal{P}^{\Delta_{k}} \beta(\eta) & \text { for } k \geq 1\end{cases}
$$

In particular, we see that

$$
d_{2 p(p-1)+1}\left(\mathcal{P}^{1} \beta(\eta) \otimes x_{t}^{p-1}\right)=\beta \mathcal{P}^{\Delta_{1}} \beta(\eta)
$$

in the $E_{2 p(p-1)+1}$-term. Since $H^{1}\left(Y_{t}\right)=0, d_{2 p+1}\left(z_{k}\right)=0$ for $1 \leq k \leq m+n$. If $d_{2 p+1}\left(y_{j}\right) \neq 0$, then we can replace the generator $y_{j}$ so that $d_{2 p+1}\left(y_{j}\right)=0$ for $1 \leq j \leq n$. In fact, by Proposition 3.10, we can write

$$
d_{2 p+1}\left(y_{j}\right)=\mathcal{P}^{1} \beta(\eta) \otimes \sum_{s=0}^{p-1} b_{s} x_{t}^{s},
$$

where $b_{s}$ are polynomials of generators of $H^{*}\left(Y_{t}\right)$ other than $x_{t}$ for $0 \leq s \leq$ $p-1$. If we put $\bar{y}_{j}$ as

$$
\bar{y}_{j}=y_{j}-\sum_{s=0}^{p-2} \frac{1}{s+1} b_{s} x_{t}^{s+1},
$$

then by (4.5), $d_{2 p+1}\left(\bar{y}_{j}\right)=\mathcal{P}^{1} \beta(\eta) \otimes b_{p-1} x_{t}^{p-1}$, and applying the differential $d_{2 p(p-1)+1}$ to $\left\{\mathcal{P}^{1} \beta(\eta) \otimes b_{p-1} x_{t}^{p-1}\right\}=0$ in the $E_{2 p(p-1)+1}$-term, we have that $\left\{\beta \mathcal{P}^{\Delta_{1}} \beta(\eta) \otimes b_{p-1}\right\}=0$. This implies that $b_{p-1}=0$, and so $d_{2 p+1}\left(\bar{y}_{j}\right)=0$. Similarly, we can replace the generators $w_{l}$ so that $d_{2 p+1}\left(w_{l}\right)=0$ for $1 \leq$ $l \leq n$. Then the $E_{2 p+2}$-term of the spectral sequence is given as

$$
E_{2 p+2}^{*, *} \cong A_{2 p+2} \otimes B_{2 p+2} \otimes \Lambda\left(\mathcal{P}^{1} \beta(\eta) \otimes x_{t}^{p-1}\right),
$$

where $A_{2 p+2} \cong A_{2 p+1} /\left(\mathcal{P}^{1} \beta(\eta)\right)$ and $B_{2 p+2}$ is generated by the generators of $B_{2 p+1}$ other than $x_{t}$. 
By (4.5), we have that

$$
d_{2 p+2}\left(z_{k}\right)= \begin{cases}\beta \mathcal{P}^{1} \beta(\eta) & \text { for } k=t, \\ 0 & \text { for } t+1 \leq k \leq m,\end{cases}
$$

and for $m+1 \leq k \leq m+n$, if $d_{2 p+2}\left(z_{k}\right) \neq 0$, then by Proposition 3.10, $d_{2 p+2}\left(z_{k}\right)=a_{k} \beta \mathcal{P}^{1} \beta(\eta)$ for some $a_{k} \in \mathbb{Z} / p$. If we set $\bar{z}_{k}=z_{k}-a_{k} z_{t}$, then $d_{2 p+2}\left(\bar{z}_{k}\right)=0$. If $d_{2 p+2}\left(y_{j}\right) \neq 0$, then by Proposition 3.10 , we can write that

$$
d_{2 p+2}\left(y_{j}\right)=\beta \mathcal{P}^{1} \beta(\eta) \otimes\left(b_{0}+b_{1} z_{t}\right),
$$

where $b_{s}$ are polynomials of generators of $B_{2 p+2}$ other than $z_{t}$ for $s=0,1$. If we set $\bar{y}_{j}=y_{j}-b_{0} z_{t}$, then $d_{2 p+2}\left(\bar{y}_{j}\right)=\beta \mathcal{P}^{1} \beta(\eta) \otimes b_{1} z_{t}$, and applying $d_{2 p+2}$ to $d_{2 p+2}\left(\bar{y}_{j}\right)$, we have that $\left(\beta \mathcal{P}^{1} \beta(\eta)\right)^{2} \otimes b_{1}=0$, which implies that $b_{1}=0$, and so $d_{2 p+2}\left(\bar{y}_{j}\right)=0$. By the same arguments, we can replace the generators $w_{l}$ so that $d_{2 p+2}\left(w_{l}\right)=0$ for $1 \leq l \leq n$. Then we obtain that

$$
E_{2 p+3}^{*, *} \cong A_{2 p+3} \otimes B_{2 p+3} \otimes \Lambda\left(\mathcal{P}^{1} \beta(\eta) \otimes x_{t}^{p-1}\right),
$$

where $A_{2 p+3} \cong A_{2 p+2} /\left(\beta \mathcal{P}^{1} \beta(\eta)\right)$ and $B_{2 p+3}$ is generated by the generators of $B_{2 p+2}$ other than $z_{t}$. For the dimensional reason and by Remark 3.12, $E_{2 p(p-1)+1}^{*, *} \cong E_{2 p+3}^{*, *}$, and by $(4.7), E_{2 p(p-1)+2}^{*, *} \cong A_{2 p(p-1)+2} \otimes B_{2 p(p-1)+2}$, where $A_{2 p(p-1)+2} \cong A_{2 p(p-1)+1} /\left(\beta \mathcal{P}^{\Delta_{1}} \beta(\eta)\right)$ and $B_{2 p(p-1)+2} \cong B_{2 p(p-1)+1}$. Furthermore, for the dimensional reason and by Remark 3.12, $E_{2 p^{2}+1}^{*, *} \cong$ $E_{2 p(p-1)+2}^{*, *}$, and so we conclude that

$$
E_{2 p^{2}+1}^{*, *} \cong A_{2 p^{2}+1} \otimes B_{2 p^{2}+1}
$$

for

$$
A_{2 p^{2}+1} \cong H^{*}\left(B^{2} \mathbb{Z} / p\right) /\left(\beta \mathcal{P}^{1} \beta(\eta), \mathcal{P}^{1} \beta(\eta)\right)
$$

and

$$
\begin{aligned}
B_{2 p^{2}+1} \cong & \mathbb{Z} / p\left[x_{t}^{p}, x_{t+1}, \ldots, x_{m}, y_{1}, \ldots, y_{n}\right] \\
& \otimes \Lambda\left(v_{1}, \ldots, v_{t-1}, z_{t+1}, \ldots, z_{m+n}, w_{1}, \ldots, w_{n}\right) .
\end{aligned}
$$

By iterating this process, we can compute the spectral sequence. In particular, the differentials are completely determined by (4.6), and so we have that for $k \geq 1$,

$$
E_{2 p^{k}+1}^{*, *} \cong A_{2 p^{k}+1} \otimes B_{2 p^{k}+1}
$$

where

$$
A_{2 p^{k}+1} \cong H^{*}\left(B^{2} \mathbb{Z} / p\right) /\left(\beta \mathcal{P}^{\Delta_{j}} \beta(\eta), \mathcal{P}^{\Delta_{j}} \beta(\eta) \mid 0 \leq j \leq k-2\right)
$$


and

$$
\begin{aligned}
B_{2 p^{k}+1} \cong & \mathbb{Z} / p\left[x_{t}^{p^{k-1}}, x_{t+1}, \ldots, x_{m}, y_{1}, \ldots, y_{n}\right] \\
& \otimes \Lambda\left(v_{1}, \ldots, v_{t-1}, z_{t+1}, \ldots, z_{m+n}, w_{1}, \ldots, w_{n}\right) .
\end{aligned}
$$

This implies that $H^{*}\left(E_{1}\right) \cong C_{t}$ as algebras, where $u$ and $v_{t}$ represent the generators $\eta$ and $\beta(\eta)$ in $H^{*}\left(B^{2} \mathbb{Z} / p\right)$. Since

$$
\begin{cases}\kappa^{*}\left(x_{i}\right)=x_{i} & \text { for } t+1 \leq i \leq m, \\ \kappa^{*}\left(y_{j}\right)=y_{j} & \text { for } 1 \leq j \leq n, \\ \kappa^{*}\left(z_{k}\right)=z_{k} & \text { for } t+1 \leq k \leq m, \\ \kappa^{*}\left(z_{k}\right)=z_{k}-a_{k} z_{t} & \text { for } m+1 \leq k \leq m+n, a_{k} \in \mathbb{Z} / p, \\ \kappa^{*}\left(w_{l}\right)=w_{l} & \text { for } 1 \leq l \leq n\end{cases}
$$

up to decomposable elements and $\mathcal{P}^{p}\left(z_{t}\right)=0$, we can take the generators of $H^{*}\left(E_{1}\right)$ satisfying the condition (4.3) with $\lambda=1$.

Next we apply same arguments to the $H$-space $E_{1}$. For the map $g$ of Lemma 4.2, a result of Lannes [11, Thm. 3.1.1] implies that there is a map $\psi_{1}: B \mathbb{Z} / p \rightarrow E_{1}$ such that $\psi_{1}^{*}=g$. The evaluation map $e_{\psi_{1}}$ : $\operatorname{Map}\left(B \mathbb{Z} / p, E_{1}\right)_{\psi_{1}} \rightarrow E_{1}$ is a homotopy equivalence by Theorem 3.1 and Proposition 3.2. Let $\iota_{1}: B \mathbb{Z} / p \rightarrow \operatorname{Map}\left(B \mathbb{Z} / p, E_{1}\right)_{\psi_{1}}$ be the adjoint of $\psi_{1} \mu$. Then, we have the following $H$-fibration by the same construction as above:

$$
E_{1} \stackrel{\simeq}{\longleftarrow} \operatorname{Map}\left(B \mathbb{Z} / p, E_{1}\right)_{\psi_{1}} \stackrel{\kappa_{1}}{\longrightarrow} E_{2} \longrightarrow B^{2} \mathbb{Z} / p,
$$

where $E_{2}=\left(\operatorname{Map}\left(B \mathbb{Z} / p, E_{1}\right)_{\psi_{1}}\right)_{h B \mathbb{Z} / p}$ denotes the Borel construction. Computing the spectral sequence for this fibration as above, we conclude that $H^{*}\left(E_{2}\right) \cong C_{t}$ with the operations (4.3) with $\lambda=0$.

Iterating this process, we have the following sequence of $H$-spaces and $H$-maps:

$$
Y_{t} \stackrel{\kappa}{\longrightarrow} E_{1} \stackrel{\kappa_{1}}{\longrightarrow} E_{2} \stackrel{\kappa_{2}}{\longrightarrow} \cdots
$$

satisfying $H^{*}\left(Y_{t}\right) \cong K_{t}, H^{*}\left(E_{s}\right) \cong C_{t}$ with the operations (4.3) with $\lambda=1$ for $s=1$ and $\lambda=0$ for $s>1, \kappa_{s}^{*}(u)=0$ and

$$
\kappa_{s}^{*}: H^{*}\left(E_{s+1}\right) /(u) \longrightarrow H^{*}\left(E_{s}\right) /(u)
$$

is an isomorphism for $s \geq 1$.

If we set $Y_{t+1}=\varliminf_{s} E_{s}$, then there is the Milnor exact sequence

$$
0 \rightarrow \varliminf_{\lim _{s}^{1}}^{1} H^{*+1}\left(E_{s}\right) \rightarrow H^{*}\left(Y_{t+1}\right) \rightarrow \varliminf_{s} H^{*}\left(E_{s}\right) \rightarrow 0 .
$$

Since $\lim _{s}^{1} H^{*+1}\left(E_{s}\right)=0$ by the Mittag-Leffler condition, we have that $H^{*}\left(Y_{t+1}\right) \cong \lim _{s} H^{*}\left(E_{s}\right) \cong K_{t+1}$, and by Proposition 3.6, we see that $Y_{t+1}$ has an $H$-structure. Let $F$ be the homotopy fiber of the composite $E_{1} \rightarrow Y_{t+1}$, then $H^{*}(F) \cong H^{*}(K(\mathbb{Z}, 2))$ by the spectral sequence argument, and this implies that $F \simeq K(\mathbb{Z}, 2)$. By the cohomology, $E_{1}$ is homotopy 
equivalent to the homotopy fiber of $[p] v_{t}: Y_{t+1} \rightarrow K(\mathbb{Z}, 3)$. Therefore, we have the following commutative diagram of fibrations:

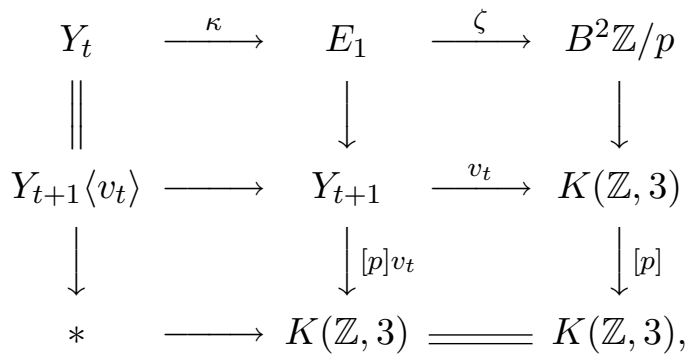

which implies that $Y_{t} \simeq Y_{t+1}\left\langle v_{t}\right\rangle$, where $Y_{t+1}\left\langle v_{t}\right\rangle$ denotes the homotopy fiber of the map $v_{t}: Y_{t+1} \rightarrow K(\mathbb{Z}, 3)$. By the induction hypothesis, $Y_{t}\langle 3\rangle \simeq Y$, and so we have that $Y_{t+1}\langle 3\rangle \simeq\left(Y_{t+1}\left\langle v_{t}\right\rangle\right)\langle 3\rangle \simeq Y$. This completes the proof.

Next, for $1 \leq t \leq n+1$, we set an algebra $L_{t}$ as

$$
\begin{aligned}
L_{t}= & \mathbb{Z} / p\left[y_{t}, \ldots, y_{n}\right] \\
& \otimes \Lambda\left(v_{1}, \ldots, v_{m+t-1}, z_{m+t}, \ldots, z_{m+n}, c_{1}, \ldots, c_{t-1}, w_{t}, \ldots, w_{n}\right)
\end{aligned}
$$

with $y_{j}, z_{k}$ and $w_{l}$ are as in $A,\left|v_{q}\right|=3$ for $1 \leq q \leq m+t-1$, and $\left|c_{r}\right|=2 p+1$ for $1 \leq r \leq t-1$. Then we have the following proposition:

Proposition 4.8. For $1 \leq t \leq n+1$, there is a $\bmod p H$-space $Z_{t}$ such that $Z_{t}\langle 3\rangle \simeq Y$ and $H^{*}\left(Z_{t}\right) \cong L_{t}$ with the following operations:

$$
\begin{cases}\beta\left(y_{j}\right)=w_{j} & \text { for } t \leq j \leq n, \\ \mathcal{P}^{1}\left(v_{m+r}\right)=c_{r} & \text { for } 1 \leq r \leq t-1, \\ \mathcal{P}^{p}\left(z_{m+l}\right)=w_{l}+\delta_{l} & \text { for } t \leq l \leq n,\end{cases}
$$

where $\delta_{l}$ is some decomposable element of $L_{t}$ for $t \leq l \leq n$.

Proposition 4.8 is proved by same arguments as in Proposition 4.1, and so we give an outline of the proof.

We proceed by an induction on $1 \leq t \leq n+1$. For $t=1$, if we set $Z_{1}=Y_{m+1}$, then by Proposition 4.1, $Z_{1}$ satisfies the required properties. We assume that there exists an $H$-space $Z_{t}$ with the conditions of Proposition 4.8, and construct an $H$-space $Z_{t+1}$ satisfying the required properties.

We can construct a $\mathcal{K}$-Hopf algebra map $h: H^{*}\left(Z_{t}\right) \rightarrow H^{*}(B \mathbb{Z} / p)$ such that $h\left(y_{t}\right)=\omega^{p^{2}}$ and $h=0$ on the other generators. By a result of Lannes, there is an $H$-map $\xi: B \mathbb{Z} / p \rightarrow Z_{t}$ such that $\xi^{*}=h$, and we see that the evaluation map $e_{\xi}: \operatorname{Map}\left(B \mathbb{Z} / p, Z_{t}\right)_{\xi} \rightarrow Z_{t}$ becomes a homotopy equivalence. For an $H$-structure $\mu$ of $B \mathbb{Z} / p$, if $\iota: B \mathbb{Z} / p \rightarrow \operatorname{Map}\left(B \mathbb{Z} / p, Z_{t}\right)_{\xi}$ denotes the adjoint of $\xi \mu$, then we have the following fibration:

$$
Z_{t} \stackrel{\simeq}{ } \operatorname{Map}\left(B \mathbb{Z} / p, Z_{t}\right)_{\xi} \longrightarrow F_{1} \longrightarrow B^{2} \mathbb{Z} / p,
$$


where $F_{1}$ is an $H$-space given by the Borel construction for $\iota$.

For $1 \leq t \leq n+1$, we set an algebra $D_{t}$ as

$$
\begin{aligned}
D_{t}= & \mathbb{Z} / p\left[u, y_{t+1}, \ldots, y_{n}\right] \\
& \otimes \Lambda\left(v_{1}, \ldots, v_{m+t}, z_{m+t+1}, \ldots, z_{m+n}, c_{1}, \ldots, c_{t}, w_{t+1}, \ldots, w_{n}\right)
\end{aligned}
$$

with $y_{j}, z_{k}, w_{l}$ and $v_{q}$ for $1 \leq q \leq m+t-1$ are as in $L_{t},|u|=2$ and $\left|v_{m+t}\right|=3$. Then, using the Serre spectral sequence, we have that $H^{*}\left(F_{1}\right) \cong D_{t}$ with the operations (4.9) and $\beta(u)=v_{m+t}$. Iterating this process, we have a sequence of $H$-spaces and $H$-maps

$$
Z_{t} \longrightarrow F_{1} \longrightarrow F_{2} \longrightarrow \cdots
$$

such that $H^{*}\left(Z_{t}\right) \cong L_{t}, H^{*}\left(F_{s}\right) \cong D_{t}$ with the operations (4.9). If we set $Z_{t+1}=\lim _{s} F_{s}$, then $Z_{t+1}$ has an $H$-structure, and using the Milnor exact sequence, we obtain that $H^{*}\left(Z_{t+1}\right) \cong L_{t+1}$ with the operations (4.9). We can show that the homotopy fiber $Z_{t+1}\left\langle v_{m+t}\right\rangle \simeq Z_{t}$, and so by the induction hypothesis, the three-connected cover $Z_{t+1}\langle 3\rangle \simeq Y$. This establishes the proof of Proposition 4.8.

Now we set $W=Z_{n+1}$. Then $W$ is a simply connected $\bmod p$ finite $H$-space such that

$$
H^{*}(W) \cong \Lambda\left(v_{1}, \ldots, v_{m+n}, c_{1}, \ldots, c_{n}\right)
$$

with $\mathcal{P}^{1}\left(z_{m+r}\right)=c_{r}$ for $1 \leq r \leq n$, and $Y \simeq W\langle 3\rangle$ by Proposition 4.8. This completes the proof of Proposition 2.6.

\section{References}

[1] J. Aguadé and L. Smith, On the mod $p$ Torus Theorem of John Hubbuck, Math. Z., 191 (1986), 325-326.

[2] A. Bousfield and D. Kan, Homotopy Limits, Completions and Localizations, Springer Lecture Notes in Math., 304 (1972).

[3] C. Broto and J.A. Crespo, H-spaces with Noetherian mod two cohomology algebra, preprint.

[4] W. Browder, Homotopy commutative H-spaces, Ann. of Math., 75 (1962), 283-311.

[5] _ On differential Hopf algebras, Trans. Amer. Math. Soc., 107 (1963), 153-178.

[6] E. Dror Farjoun, Cellular Spaces, Null Spaces and Homotopy Lacalization, Springer Lecture Notes in Math., 1622 (1996).

[7] W.G. Dwyer and C.W. Wilkerson, Spaces of null homotopic maps, Astérisque, 191 (1990), 97-108.

[8] Y. Hemmi, Higher homotopy commutativity of $H$-spaces and the mod $p$ Torus theorem, Pacific J. Math., 149 (1991), 95-111.

[9] K. Iriye and A. Kono, Mod $p$ retracts of G-product spaces, Math. Z., 190 (1985), 357-363.

[10] D. Kraines, The kernel of the loop suspension map, Illinois J. Math., 21 (1977), 91-108. 
[11] J. Lannes, Sur les espaces fonctionnels dont la source est le classifiant d'un p-groupe abélien élémentaire, Publ. Math. Inst. HES, 75 (1992), 135-244.

[12] J.P. Lin, Loops of $H$-spaces with finitely generated cohomology rings, Topology Appl., 60 (1994), 131-152.

[13] _ Finitely generated cohomology Hopf algebras and torsion, Pacific J. Math., 172 (1996), 215-221.

[14] C. McGibbon, Homotopy commutativity in localized groups, Amer. J. Math., 106 (1984), 665-687.

[15] _ Higher forms of homotopy commutativity and finite loop spaces, Math. Z., 201 (1989), 363-374.

[16] J. Milnor and J.C. Moore, On the structure of Hopf algebras, Ann. of Math., 81 (1965), 211-264.

[17] J.A. Neisendorfer, Localizations and connected covers of finite complexes, Contemp. Math., 181 (1995), 385-390.

[18] M. Slack, A classification theorem for homotopy commutative mod $2 \mathrm{H}$-spaces with finitely generated cohomology rings, Mem. Amer. Math. Soc., 92 (1991).

[19] J.D. Stasheff, Homotopy associativity of H-spaces, I, II, Trans. Amer. Math. Soc., 108 (1963), 275-292, 293-312.

[20] Z. Wojtkowiak, On maps from holim F to Z, Springer Lecture Notes in Math., 1298 (1986), 227-236.

[21] A. Zabrodsky, On phantom maps and a theorem of H. Miller, Israel J. Math., 58 (1987), 129-143.

Received January 2, 1998 and revised August 5, 1998. The author was partially supported by JSPS Research Fellowships for Young Scientists.

HiRoshima UNIVERSITY

KagAMIYAMA $1-3-1$

Higashi-Hiroshima 739-8526

JAPAN

E-mail address: yusuke@top3.math.sci.hiroshima-u.ac.jp 